\title{
35 años de cambio en densidad y biomasa del megazoobentos del golfo de Batabanó, Cuba, e implicaciones para la langosta, Panulirus argus (Decapoda: Palinuridae)
}

\author{
Alexander Lopeztegui Castillo ${ }^{1,2^{*}} \&$ Diana Martínez Coello ${ }^{2}$ \\ 1. Instituto Politécnico Nacional, Centro Interdisciplinario de Ciencias Marinas. Av. Instituto Politécnico Nacional s/n, \\ Col. Playa Palo de Santa Rita, La Paz, BCS, C.P. 23096, La Paz, BCS, México; alopeztegui@yahoo.com \\ 2. Centro de Investigaciones Pesqueras, Ministerio de la Industria Alimentaria, Calle 246, No. 503 entre 5ta. Avenida y \\ Mar, Barlovento, C.P. 11300, Playa, La Habana, Cuba; dianita.mcoello@gmail.com \\ * Correspondencia
}

Recibido 25-III-2020. Corregido 31-VII-2020. Aceptado 11-IX-2020.

\begin{abstract}
Gulf of Batabanó, Cuba, and implications for the lobster, Panulirus argus (Decapoda: Palinuridae). Introduction: Benthos is an efficient indicator of environmental disturbances, biodiversity and trophic dynamics. In Cuba, benthic mollusks have been reported as the main food for the lobster Panulirus argus. Objectives: To estimate spatiotemporal variations in the soft-bottom benthic community of the Gulf of Batabanó, and to infer, with emphasis on mollusks, the effect of such variations on the availability of food for spiny lobsters. Methods: A historical analysis of the variations in the density and biomass of megazoobenthos (organisms $\geq 4 \mathrm{~mm}$ ) and particularly of megazoobenthic mollusks was carried out, taking as a reference the studies published in 1990 (with samples from 1983) and recent data (2007-2018) recorded on site using comparable methodologies based on sediment drags. Abundance was given as density (ind $/ \mathrm{m}^{2}$ ) and biomass in $\mathrm{g} / \mathrm{m}^{2}$. Results: It was found that both the density and the biomass were significantly higher at the end of the last century and show a tendency to gradually decrease towards recent times. Regarding the values reported in 1983, the density of organisms showed a decrease of $73 \%$, however, the biomass experienced an even greater decrease (86\%). The areas with the highest density and biomass, both benthos and mollusks, have varied from 1983 and are currently located towards the center of the gulf. Despite this, bivalves continue to be better represented than gastropods. In general, mollusks showed a significant decrease (55\% and $88 \%$ respectively). Conclusions: The deterioration of the benthic community in the fishing areas of the Gulf of Batabanó was corroborated and it was demonstrated, based on megazoobenthos, that malacofauna can be used as a reliable indicator of the general parameters of the softbottom benthic community. The availability of food for lobsters have decreased $55.6 \%$ compared to 1983 . This could help to explain the current changes in the abundance, distribution and nutritional condition of lobsters, which could also be related to the recent megazoobenthos variations.
\end{abstract}

Key words: food availability; benthos; mollusks; soft bottoms; spiny lobster.

Lopeztegui Castillo, A., \& Martínez Coello, D. (2020). 35 años de cambio en densidad y biomasa del megazoobentos del golfo de Batabanó, Cuba, e implicaciones para la langosta, Panulirus argus (Decapoda: Palinuridae). Revista de Biología Tropical, 68(4), 1346-1356.

Los atributos de la comunidad bentónica han sido con frecuencia utilizados para estimar la calidad del hábitat y el nivel de impacto ambiental en varias regiones del mundo (Çinar et al., 2015; McLaverty et al., 2020). Para el estudio del zoobentos se han establecido cuatro grupos fundamentales a partir del tamaño de los organismos: microbentos, meiobentos, macrobentos y megabentos (Sakshaug, HelgeJohnsen, \& Kovacs, 2009). Aunque existen 
múltiples criterios acerca de los límites de tamaño definidos para cada categoría, la repercusión ecológica de cada grupo es única y está objetivamente determinada. Respecto a los organismos mayores de 4 milímetros, clasificados como megazoobentos (Alcolado, 1990; Alcolado et al., 1998; Sakshaug et al., 2009), se ha demostrado que tienen una relevante función como bioindicadores de contaminación ambiental, impactos antrópicos y biodiversidad (Alcolado, \& Espinosa, 1996).

También se ha hecho notar la importancia del megazoobentos como componente de la trama trófica y alimento esencial para varias especies comerciales entre las que destacan las langostas (Colinas-Sánchez, \& Briones-Fourzán, 1990; Espinosa et al., 1990). A nivel mundial, y particularmente en Cuba, los moluscos bentónicos han sido reportados como componentes esenciales en la dieta de varias langostas espinosas entre las que se encuentra Panulirus argus (Latreille, 1084), principal recurso pesquero de Cuba y uno de los productos más apreciados en el mercado internacional (Herrera et al., 1991; Puga-Millán et al., 2013; Puga-Millán et al., 2018).

La mayor parte de los estudios de bentos en Cuba implican sólo componentes de la macro o meiofauna (Arias-Scheirber et al., 2008; Armenteros, Williams, Creagh, \& CapetilloPiñar, 2008; Hidalgo, Toledo, \& GranadosBarba, 2015). Respecto al megazoobentos de fondos blandos un menor número de estudios se ha desarrollado, por lo que el tema requiere de estudios que aporten información acerca del empleo de sus propiedades para, como han sugerido Labra, Hernández-Miranda y Quiñones (2020), inferir cambios en las condiciones del hábitat y el flujo de energía trófica en los ecosistemas. El golfo de Batabanó, región pesquera más importante de Cuba (Cruz et al., 1990; Baisre, 2018), ha sido de las pocas en la que se han desarrollado investigaciones que incluyen al megazoobentos, siendo el trabajo de Alcolado (1990) la principal obra de referencia.

El interés pesquero en el golfo de Batabanó ha motivado el estudio de procesos de elevada repercusión ecológica, siendo numerosas las investigaciones que describen significativos cambios en la flora y fauna bentónica (Arias-Scheiber et al., 2008; Cerdeira-Estrada, Lorenzo-Sánchez, Areces-Mallea, \& MartínezBayón, 2008). El escenario actual, que evidencia el decrecimiento de las capturas de múltiples recursos entre los que destaca $P$. argus, le confiere aún mayor relevancia a este tipo de estudios (Baisre, 2018; Puga-Millán et al., 2018). En este contexto, fue objetivo de la presente investigación, determinar variaciones espacio-temporales en la densidad y biomasa del megazoobentos de los fondos blandos del golfo de Batabanó, e inferir, haciendo énfasis en los moluscos, el efecto de tales variaciones en la disponibilidad de alimento para las langostas.

\section{MATERIALES Y MÉTODOS}

El presente estudio se realizó en los fondos blandos del golfo de Batabanó, en el suroeste del Cuba, en el Mar Caribe, considerado una extensa macrolaguna con $6 \mathrm{~m}$ de profundidad media y predominio de fondos blandos cubiertos de pastos marinos (Thalassia testudinum Banks ex König) de densidad entre media y baja (Alcolado, 1990). Se registraron datos del 2007, 2008, 2009, 2010, 2015, 2017 y 2018, tratados como "período 2007-2018", en 12 de los 15 sitios muestreados por Alcolado (1990) en 1983. Además, se utilizaron los datos de 1983 como referencia para realizar comparaciones de densidad y biomasa de los organismos con los obtenidos en el presente estudio (Alcolado, 1990) (Fig. 1).

Los muestreos se realizaron mediante arrastres (tres réplicas por sitio). La metodología seguida tanto para el muestreo en 1983 como para el presente estudio se especifica en Alcolado (1990). La rastra utilizada en el período 2007-2018, que posee en su entrada un área de $56 \times 25 \mathrm{~cm}^{2}$, no fue la misma empleada en 1983, pero ambas presentan similitudes estructurales y funcionales que hacen comparables los resultados siempre que la abundancia se estandarice como densidad de organismos 


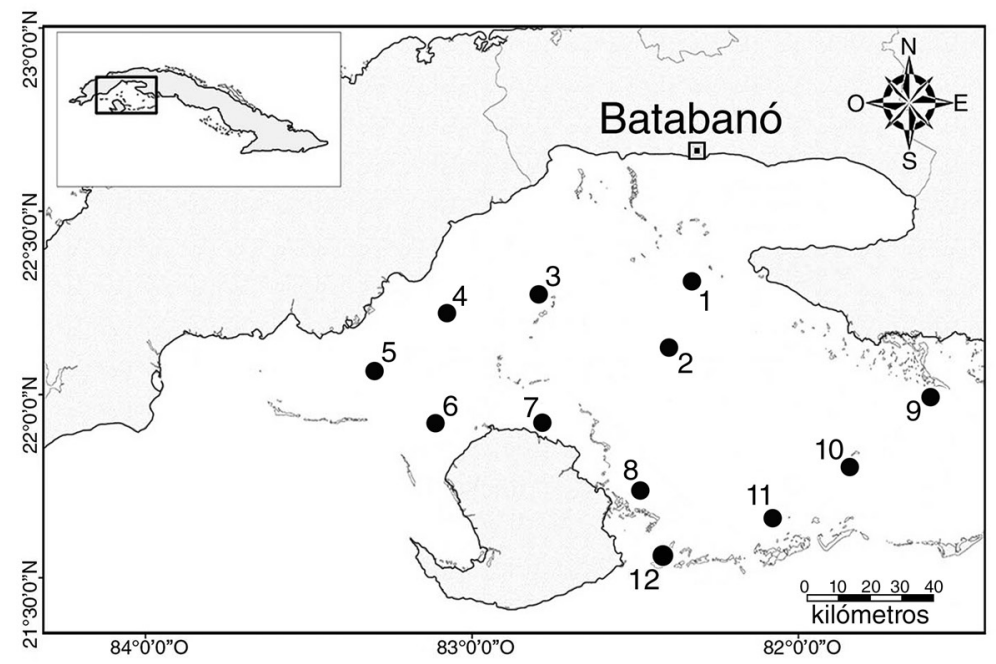

Fig. 1. Ubicación de los sitios de muestreo para colecta de megazoobentos (excluyendo esponjas, corales y gorgonáceos) en los fondos blandos del golfo de Batabanó, Cuba. Las estaciones representadas pertenecen al período 2007-2018.

Fig. 1. Location of the sampling sites for the collection of megazoobenthos (excluding sponges, corals and gorgonians) in the soft bottoms of the Gulf of Batabanó, Cuba. The stations represented belong to the 2007-2018 period.

(ind $\left./ \mathrm{m}^{2}\right)$ y la biomasa se exprese en unidades de área $\left(\mathrm{g} / \mathrm{m}^{2}\right)$. En 2007-2018 cada arrastre (réplica) tuvo una duración promedio de $40 \mathrm{~s}$, en los que el barco se movió a 2 nudos, para un área total arrastrada de $21 \mathrm{~m}^{2}$. De 2007 a 2015 se utilizaron sólo como valor anual de densidad y biomasa debido a que no se cuenta con los datos respectivos de cada sitio. En 2017 y 2018 fue posible realizar un muestreo pormenorizado, por sitios, de ambas variables, lo que permitió un análisis espacial más detallado y la comparación por Clases (Gastropoda y Bivalvia) de los moluscos encontrados.

La fauna recolectada (organismos vivos excluyendo esponjas, corales y gorgonáceos, que no han sido reportados en la dieta de $P$. argus en Cuba) se seleccionó en un tamiz de $4 \mathrm{~mm}$, se almacenó en formaldehido neutralizado al $10 \%$ y se empleó para determinar los valores de densidad (ind $\left./ \mathrm{m}^{2}\right)$ y biomasa $\left(\mathrm{g} / \mathrm{m}^{2}\right)$ a partir del total de organismos, su peso y el área total $\left(21 \mathrm{~m}^{2}\right)$ de cada réplica. La biomasa (peso seco), se determinó mediante una balanza técnica de $0.01 \mathrm{~g}$ de precisión. Mediante microscopía estereoscópica se identificaron los organismos, hasta nivel de especie en caso de los moluscos y hasta el menor taxón posible los restantes. La identificación de moluscos se basó en las descripciones de Mikkelsen, y Bieler (2007) y Redfern (2013). El resto de los grupos se identificaron según criterios generales (Alcolado, 1990) y el apoyo de especialistas en comunidades bentónicas de la plataforma cubana.

Para los análisis, los organismos megazoobentónicos se separaron en dos grupos: moluscos (alimento principal de $P$. argus) y bentos (todos los organismos excluyendo moluscos). A falta de un sistema de clasificación que establezca un límite de tamaño superior para el megazoobentos, y asumiendo que, según el criterio de los autores, es poco probable que las langostas depreden organismos cuyo tamaño exceda los $6 \mathrm{~cm}$ de diámetro menor (cinco veces el tamaño de la boca de una langosta adulta), se contabilizaron sólo organismos menores a $6 \mathrm{~cm}$.

Las variaciones en el potencial alimentario disponible para las langostas son una de las principales implicaciones de los cambios en la densidad y biomasa de bentos. Por esta razón, los datos del 2017 y 2018 se emplearon además para estimar la disminución del potencial alimentario a partir de los valores 
de densidad y biomasa de moluscos reportados por (Alcolado, 1990) en 1983. Ante la carencia de un índice que cuantifique la disponibilidad de alimento para $P$. argus, la variación en el potencial alimentario se estimó a partir del promedio de los porcentajes en que disminuyeron (entre 1983 y 2018) la densidad y la biomasa de moluscos, menos el $20 \%$ de la disminución de otros grupos. Esta cantidad se definió basado en que, según Herrera et al. (1991), los moluscos constituyen el $80 \%$ de la dieta de las langostas que se alimentan en el seibadal, por lo que otros grupos integrantes del megazoobentos sólo conforman el $20 \%$ del alimento disponible.

Procesamiento estadístico: Mediante la prueba de Kolmogorov-Smirnov se comprobó que los datos no cumplen con las premisas de una distribución normal, por lo que se aplicó estadística no paramétrica con un nivel de significación de $\mathrm{P}<0.05$. Las comparaciones pareadas entre años y entre grupos taxonómicos se efectuaron mediante la prueba $U$ de Mann-Whitney. Las comparaciones múltiples entre años y entre sitios se realizaron aplicando la prueba Kruskall-Wallis. Para estimar si las variaciones en la densidad y biomasa de megazoobentos (sin incluir moluscos), se relacionan con la densidad y biomasa (variables dependientes) de moluscos megazoobentónicos, se aplicó un análisis de regresión lineal simple. Para determinar la tendencia en ambas variables (densidad y biomasa), se construyó un diagrama de dispersión bidimensional para cada una de ellas, con los años (1983, 2007-2018) como variable independiente (eje $\mathrm{X})$. La relación entre los puntos se describió mediante una recta.

\section{RESULTADOS}

Los valores de biomasa y densidad de organismos del megazoobentos, y de moluscos en particular, resultaron bajos en 2017 y 2018 (Tabla 1). Tanto la densidad como la biomasa de bentos resultaron similares (U de MannWhitney, $\mathrm{P}=0.744$ y $\mathrm{P}=0.489$ respectivamente) entre 2017 y 2018, hecho que también se evidenció al comparar la densidad y biomasa de moluscos megazoobentónicos (U de MannWhitney, $\mathrm{P}=0.681$ y $\mathrm{P}=0.285$ respectivamente).

TABLA 1

Biomasa y densidad del megazoobentos $(\geq 4 \mathrm{~mm}$ ) y de los moluscos con concha que forman parte de la comunidad megazoobentónica en los fondos blandos del golfo de Batabanó, Cuba

TABLE 1

Biomass and density of megazoobenthos $(\geq 4 \mathrm{~mm})$ and shell mollusks that are part of the megazoobenthic community in the soft bottoms of the Batabanó Gulf, Cuba

\begin{tabular}{|c|c|c|c|c|c|c|}
\hline \multicolumn{7}{|c|}{2017} \\
\hline & Media & Mediana & Mínimo & Máximo & V & D.E. \\
\hline Biomasa bentos $\left(\mathrm{g} / \mathrm{m}^{2}\right)$ & 5.49 & 1.84 & 0.16 & 34.70 & 59.04 & 7.68 \\
\hline Densidad bentos (ind $/ \mathrm{m}^{2}$ ) & 4.99 & 4.29 & 0.33 & 12.05 & 8.60 & 2.93 \\
\hline Biomasa moluscos $\left(\mathrm{g} / \mathrm{m}^{2}\right)$ & 0.73 & 0.26 & 0.00 & 9.74 & 2.16 & 1.47 \\
\hline Densidad moluscos (ind $/ \mathrm{m}^{2}$ ) & 1.86 & 1.31 & 0.00 & 6.52 & 2.51 & 1.58 \\
\hline \multicolumn{7}{|c|}{2018} \\
\hline Biomasa bentos $\left(\mathrm{g} / \mathrm{m}^{2}\right)$ & 4.21 & 1.35 & 0.20 & 32.87 & 47.97 & 6.93 \\
\hline Densidad bentos (ind $/ \mathrm{m}^{2}$ ) & 6.12 & 4.10 & 0.48 & 24.95 & 27.16 & 5.21 \\
\hline Biomasa moluscos $\left(\mathrm{g} / \mathrm{m}^{2}\right)$ & 0.91 & 0.35 & 0.01 & 5.46 & 1.65 & 1.29 \\
\hline Densidad moluscos (ind $/ \mathrm{m}^{2}$ ) & 2.52 & 1.41 & 0.10 & 22.00 & 12.09 & 3.48 \\
\hline
\end{tabular}

V: varianza; D.E.: desviación estándar.

V: variance; D.E.: standard deviation. 


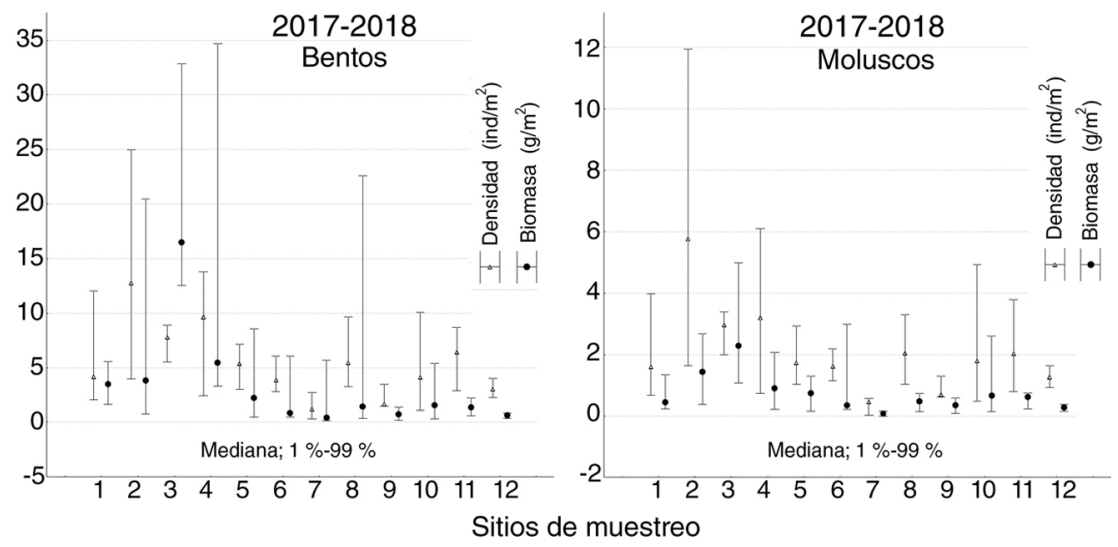

Fig. 2. Densidad y Biomasa de megazoobentos $(\geq 4 \mathrm{~mm})$ y de moluscos megazoobentónicos en cada sitio muestreado en los fondos blandos del golfo de Batabanó, Cuba (2017 y 2018).

Fig. 2. Density and Biomass of megazoobenthos $(\geq 4 \mathrm{~mm})$ and megazoobenthic mollusks at each site sampled in the soft bottoms of the Batabanó Gulf, Cuba (2017 and 2018).

El análisis espacial de la densidad promedio del 2017 y 2018, reveló que los sitios con mayores valores, tanto de megazoobentos como de moluscos megazoobentónicos, fueron 2, 3 y 4. Similar comportamiento se obtuvo también para la biomasa (Fig. 2).

Los cambios en la densidad y biomasa de megazoobentos influyeron respectiva y significativamente en la densidad y biomasa de moluscos megazoobentónicos, tanto en la escala espacial como en la temporal. Se comprobó que las variaciones espacio-temporales de la comunidad de moluscos y del bentos fueron semejantes y mostraron una significativa relación de dependencia fundamentada en el resultado de las regresiones (Tabla 2).

El promedio anual de la densidad de organismos se incrementó en 2007 respecto a 1983 pero presentó tendencia a la disminución entre 2007 y 2018. Particularmente en moluscos ( $y=$ $\left.12.3229-1.2095^{*} \mathrm{x}\right)$ esta tendencia resultó 54 $\%$ menos pronunciada que para el bentos $(\mathrm{y}=$ 22.7796-2.2346*x). En ambos grupos el mayor decrecimiento sucedió entre 2010 y 2015. La biomasa también mostró una tendencia a la disminución, con una pendiente $44 \%$ mayor

TABLA 2

Análisis de regresión entre la densidad y biomasa de megazoobentos (variables independientes) y la densidad y biomasa de moluscos megazoobentónicos (variables dependientes) en los 12 sitios muestreados en 2017 y 2018 (variación espacial) y como promedios anuales de los años estudiados $(1983 ; 2007-2018)$ durante todo el período de muestreo (variación temporal)

TABLE 2

Regression analysis between the density and biomass of megazoobenthos (independent variables) and the density and biomass of megazoobenthic mollusks (dependent variables) at twelve sampled sites in 2017 and 2018 (spatial variation), and like annual average for each sampled year (1983; 2007-2018) during all study (temporal variation)

\begin{tabular}{|c|c|c|c|c|}
\hline \multicolumn{5}{|c|}{$2017-2018$} \\
\hline & $\mathrm{R}$ & $\mathrm{R}^{2}$ & $\mathrm{R}^{2}$ ajustado & $\mathrm{P}$ \\
\hline Densidad bentos-Densidad moluscos & 0.977 & 0.954 & 0.954 & 0.001 \\
\hline Biomasa bentos-Biomasa moluscos & 0.394 & 0.155 & 0.143 & 0.001 \\
\hline \multicolumn{5}{|c|}{$1983-2018$} \\
\hline Densidad bentos-Densidad moluscos & 0.799 & 0.638 & 0.577 & 0.017 \\
\hline Biomasa bentos-Biomasa moluscos & 0.807 & 0.652 & 0.594 & 0.015 \\
\hline
\end{tabular}



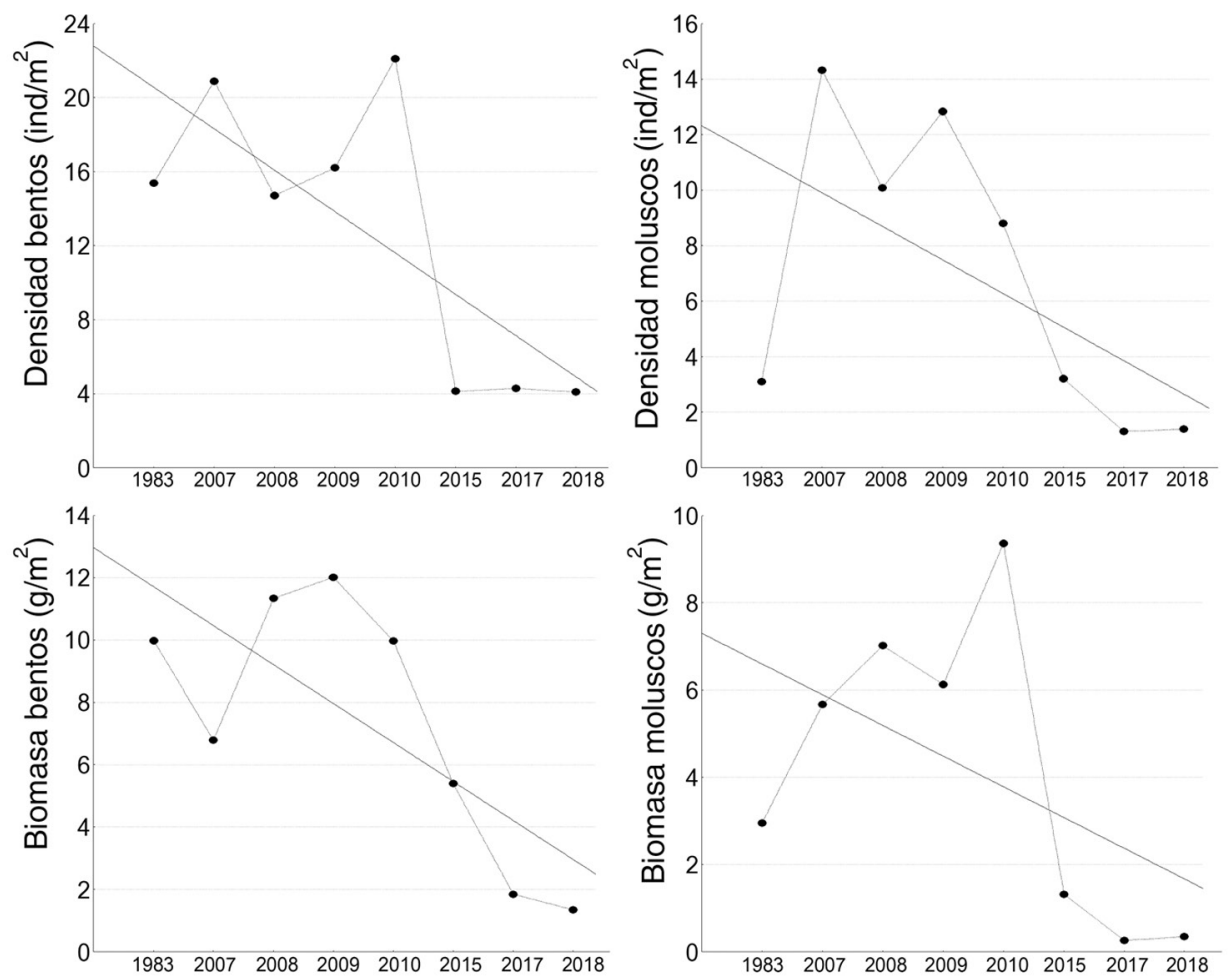

Fig. 3. Densidad y biomasa de megazoobentos y de moluscos megazoobentónicos de fondos blandos en el golfo de Batabanó, Cuba.

Fig. 3. Density and biomass of megazoobenthos and megazoobenthic soft-bottom mollusks in the Gulf of Batabanó, Cuba.

para el bentos $(\mathrm{y}=12.9575-1.25 * \mathrm{x})$ que para los moluscos $\left(\mathrm{y}=7.2996-0.7038^{*} \mathrm{x}\right)$. Los valores de biomasa en 2007 fueron mayores a los existentes en 1983 excepto para la biomasa de bentos, caso en el que la tendencia al decrecimiento se observó a partir de 2010 (Fig. 3). Respecto a 1983, en 2018 la densidad de bentos mostró una significativa $(\mathrm{P}<0.001)$ disminución del $73 \%$, sin embargo, la biomasa experimentó una disminución aún mayor (86\%). La densidad y biomasa de moluscos también mostraron un significativo decrecimiento $(\mathrm{P}<$ 0.001 en ambos casos), cuantificado en 55 y 88 $\%$ respectivamente. Con base en estos valores, entre 1983 y 2018 la disminución en el potencial alimentario disponible para las langostas se estimó en un: $[(55 \%+88 \%) / 2]-0.2[(73 \%$ $+86 \%) / 2]=55.6 \%$.

En 2017 la densidad y biomasa de bivalvos fueron superiores $(\mathrm{P}=0.001 ; \mathrm{P}=0.006$ respectivamente) a las de gastrópodos, comportamiento que también se evidenció en 2018 ( $\mathrm{P}=0.001$ y $\mathrm{P}=0.011$ respectivamente; Fig. 4).

\section{DISCUSIÓN}

Según reportó Alcolado (1990), en el período 1981-1985, los sitios con mayor densidad y biomasa de megazoobentos (excluyendo esponjas, corales y gorgonáceos), fueron 5, 6 , 8,9 y 11. Según los resultados obtenidos en el presente estudio, tales sitios no destacan entre las de mejor condición, encontrándose mayores densidades y biomasas hacia la porción central del golfo (fundamentalmente sitios 2, 3 y 4). Esto puede ser consecuencia de los cambios que, a lo largo de más de tres décadas, han tenido lugar en esta región. Como ha sido reportado, tales cambios se traducen 


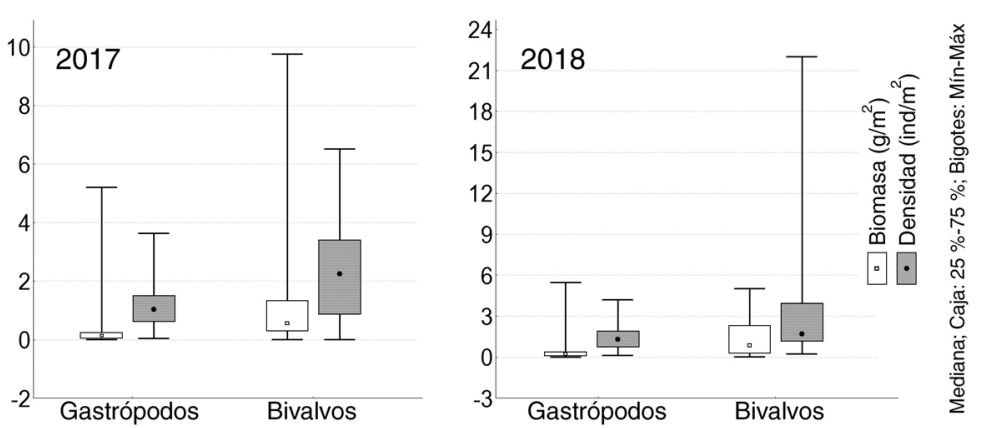

Fig. 4. Densidad y Biomasa de los bivalvos y gastrópodos encontrados como parte de la comunidad megazoobentónica ( $\geq$ $4 \mathrm{~mm}$ ) en los fondos blandos del golfo de Batabanó, Cuba.

Fig. 4. Density and Biomass of bivalves and gastropods found as part of the megazoobenthic community ( $\geq 4 \mathrm{~mm})$ in the soft bottoms of the Gulf of Batabanó, Cuba.

en el deterioro de las condiciones tanto bióticas como abióticas en las zonas pesqueras (Arias-Scheriber et al., 2008; Cerdeira-Estrada et al., 2008; Capetillo-Piñar, Villalejo-Fuerte, \& Tripp-Quezada, 2015; Betanzos-Vega, Capetillo-Piñar, Lopeztegui-Castillo, GarcésRodríguez, \& Tripp-Quezada, 2019). También se ha reportado deterioro en áreas próximas a la costa, que constituyen zonas de crianza para juveniles de varias especies de interés comercial y/o ecológico (Lopeztegui-Castillo \& Capetillo-Piñar, 2008).

Las variaciones espaciales de la densidad y la biomasa corroboraron, además, una condición de deterioro generalizada en esta región. Entre los sitios de menores valores se encontraron algunos (sitios 6 y 12) próximos al borde de la plataforma, donde el mayor intercambio con aguas profundas (del Mar Caribe) debiera favorecer la existencia de mejores condiciones para el desarrollo la fauna bentónica (MartínezDaranas, Betanzos-Vega, Lopeztegui-Castillo, \& Capetillo-Piñar, 2018). No obstante, la condición más deteriorada se obtuvo en el sitio 7 , más próximo a la costa y a la desembocadura del río Las Casas. Esto demuestra que el impacto antrópico en las zonas más próximas a las costas, muchas de las cuáles constituyen áreas de cría de especies comerciales como $P$. argus, es de elevada magnitud (Lopeztegui-Castillo \& Capetillo-Piñar, 2008). Las variaciones temporales confirmaron una tendencia gradual al decrecimiento tanto de la abundancia como de la biomasa de megazoobentos. Los valores obtenidos en 2017 y 2018 fueron inferiores a los reportados por Alcolado (1990).

Además de implicar afectaciones a la biodiversidad, el deterioro de la comunidad megazoobentónica en el golfo de Batabanó puede traer como consecuencia una disminución en la productividad pesquera (Gómez, Ibarzábal, \& Silva, 1980). La energía que ingresa a la trama trófica mediante organismos bentónicos disminuye con el decrecimiento de la densidad y la biomasa del megazoobentos. Esto afecta primera y directamente a organismos estrictamente bentófagos, como P. argus. Sin embargo, otros organismos de eslabones tróficos subsecuentes, como los depredadores de langosta, podrían llegar a ser impactados. La langosta $P$. argus es considerada un mesodepredador omnívoro que forma parte de la dieta de varias especies de interés económico o ecológico como pargos, chernas, pulpos, morenas y tiburones (Cruz et al., 1990; Briones-Fourzán et al., 2019). Estas especies podrían presentar variaciones en su crecimiento y hasta en sus patrones de abundancia y distribución, a causa de variaciones significativas en el megazoobentos que constituye alimento para las langostas. En los ecosistemas marinos, los mesodepredadores omnívoros juegan un papel determinante en la transmisión de la materia orgánica a través de la trama trófica (Long, Bruno, \& Duffy, 2011). 
Desde el año 1985 las capturas de langosta en Cuba, y particularmente en el golfo de Batabanó, han presentado una tendencia gradual al decrecimiento (Puga-Millán et al., 2013; Alzugaray-Martínez et al., 2018; Baisre, 2018). Aunque ya ha sido demostrado que tal decline tiene un origen multifactorial, sólo algunas de las causas han sido identificadas (Piñeiro-Soto, Arsenio-Areces, Puga-Milián, Cobas-Gómez, \& de León-González, 2017; Alzugaray-Martínez et al., 2018). Las investigaciones existentes permiten concluir que desde mediados de la década de 1980, las comunidades bentónicas de fondos blandos en el golfo de Batabanó han experimentado un gradual y significativo deterioro. El presente estudio cuantifica este deterioro basado fundamentalmente en organismos que constituyen alimento para las langostas, haciendo evidente que otro de los factores que influye en la disminución de las capturas, es la reducción en la disponibilidad de alimento. Según Lozano-Álvarez, \& Aramoni-Serrano (1996), el alimento puede influir determinantemente en la abundancia, distribución y condición nutricional de las langostas. Lopeztegui-Castillo, Capetillo-Piñar y Betanzos-Vega (2012), sugieren que las variaciones encontradas en la condición nutricional de $P$. argus en el golfo de Batabanó, se relacionaron con las afectaciones de la comunidad bentónica y la disminución de la disponibilidad de alimento en esta región.

El análisis de regresión practicado a los datos de 2017 y 2018, demostró que la densidad y biomasa de moluscos megazoobentónicos estuvieron relacionadas, respectivamente, con la densidad y biomasa de megazoobentos. Esta relación, estadísticamente significativa, se comprobó también al analizar los datos anuales del período en estudio (1983-2018). Consecuentemente, la densidad o biomasa de moluscos ( $>$ de $4 \mathrm{~mm}$ ) de fondos blandos, podría ser utilizada para inferir la densidad o biomasa de megazoobentos, con un grado de certidumbre que varía entre 80 y $98 \%$. Similar resultado fue obtenido por Alcolado y Espinosa (1996), quienes encontraron una relación de $\mathrm{r}=$ 0.892 entre el número de especies de moluscos bentónicos y el número total de especies del bentos. Estas relaciones posibilitan estimar el estado de la comunidad bentónica a partir del estudio de sólo uno de sus componentes, los moluscos, relevantes además por su importancia como grupo bioindicador y como componentes de la trama trófica (alimento esencial para las langostas).

Dentro de los moluscos, los bivalvos ocuparon el mayor porcentaje, con mayores valores de densidad y biomasa en 2017 y 2018 . Esta tendencia ya había sido reportada por Alcolado (1990) y parece ser una de las características de la comunidad de moluscos que no ha sufrido considerables variaciones, sugiriendo que los factores que determinan el deterioro del bentos en el golfo de Batabanó, impactan de manera similar a ambos grupos de moluscos. Ecológicamente, esto pudiera ser expresión de resiliencia y significar que la encontrada proporción favorece el equilibrio dinámico con el ambiente y resulta positiva para esta comunidad. Sin embargo, los gastrópodos han resultado ser más importantes que los bivalvos en la dieta de las langostas (Espinosa et al., 1990; Herrera et al., 1991; Martínez-Coello, Lopeztegui-Castillo, \& Amador-Marrero, 2015), lo cual podría deberse a que se encuentran más accesibles dado que no se entierran en el sedimento.

Varias investigaciones, empleando diferentes grupos de organismos tanto de la meiofauna como de la macrofauna, han reportado afectaciones en la comunidad bentónica del golfo de Batabanó, particularmente sobre fondos blandos (Arias-Scheirber et al., 2008; Armenteros et al., 2008; Lopeztegui-Castillo, \& Capetillo-Piñar, 2008; Armenteros et al., 2012; Hidalgo, Toledo, \& Granados-Barba, 2015). Otros estudios han mostrado resultados similares basados principalmente en cambios de biotopo y las características de la vegetación submarina (Cerdeira-Estrada et al., 2008; Martínez-Daranas, Cano-Mallo, \& Clero-Alonso, 2009; Martínez-Daranas, \& Suárez, 2018; Martínez-Daranas et al., 2018). Por primera vez basado en atributos de la comunidad megazoobentónica, esencialmente densidad y biomasa, los presentes resultados corroboran los signos 
de deterioro y la degradación del bentos, lo que resulta novedoso y aporta relevante información al entendimiento de la dinámica de los ecosistemas de esta región.

Declaración de ética: los autores declaran que todos están de acuerdo con esta publicación y que han hecho aportes que justifican su autoría; que no hay conflicto de interés de ningún tipo; y que han cumplido con todos los requisitos y procedimientos éticos y legales pertinentes. Todas las fuentes de financiamiento se detallan plena y claramente en la sección de agradecimientos. El respectivo documento legal firmado se encuentra en los archivos de la revista.

\section{AGRADECIMIENTOS}

Se desea agradecer a los trabajadores de la empresa pesquera de Batabanó, particularmente a la tripulación de las embarcaciones Plástico 10 y Traviesa, por el apoyo recibido durante los cruceros de investigación. A José Espinosa Sáez, por su vital contribución en la identificación de moluscos y otros organismos bentónicos. Especialmente se agradece el apoyo financiero recibido del CONACYT.

\section{RESUMEN}

Introducción: El bentos constituye un eficiente indicador de disturbios ambientales, de biodiversidad y de dinámica trófica. En Cuba, los moluscos bentónicos han sido reportados como el principal alimento de la langosta Panulirus argus. Objetivos: Estimar variaciones espaciotemporales en la comunidad bentónica de fondos blandos del golfo de Batabanó, e inferir, haciendo énfasis en los moluscos, el efecto de tales variaciones en la disponibilidad de alimento para las langostas. Métodos: Se realizó un análisis histórico de las variaciones en la densidad y biomasa de megazoobentos (organismos $\geq 4 \mathrm{~mm}$ ) y particularmente de moluscos megazoobentónicos, tomando como referencia los estudios realizados desde 1990 (muestreos del año 1983) y datos actuales (2007-2018) registrados in situ mediante metodologías comparables. Resultados: Se encontró que tanto la densidad como la biomasa eran significativamente mayores al final del pasado siglo y muestran tendencia a disminuir gradualmente hacia la actualidad. Respecto a los valores reportados en 1983, la densidad de organismos mostró un decrecimiento del 73
$\%$, sin embargo, la biomasa experimentó una disminución aún mayor (86\%). Las áreas de mayor densidad y biomasa, tanto de bentos como de moluscos, han variado respecto a lo reportado para 1983 y actualmente se ubican hacia el centro del golfo. A pesar de ello, los bivalvos continúan estando mejor representados que los gastrópodos. En general, los moluscos mostraron un significativo decrecimiento (55\% y $88 \%$ respectivamente). Conclusiones: Se corroboró el deterioro de la comunidad bentónica en las áreas pesqueras del golfo de Batabanó y se demostró, basado en el megazoobentos, que la malacofauna puede utilizarse como un indicador fiable de los parámetros generales de la comunidad bentónica de fondos blandos. Se estimó que la disponibilidad de alimento para las langostas ha disminuido en un $55.6 \%$ respecto a 1983 , lo cual, unido a las variaciones espaciales del megazoobentos, puede contribuir a explicar los actuales cambios en la abundancia y distribución de las langostas.

Palabras clave: Disponibilidad de alimento, bentos, moluscos, fondos blandos, langosta espinosa.

\section{REFERENCIAS}

Alcolado, P.M. (1990). El bentos de la macrolaguna del Golfo de Batabanó. La Habana, Cuba: Editorial Academia.

Alcolado, P.M., \& Espinosa, J. (1996). Empleo de las comunidades de moluscos marinos de fondos blandos como bioindicadores de la diversidad del megazoobentos y de la calidad ambiental. Iberus, 14, 79-84.

Alcolado, P.M., Espinosa, J., Martínez-Estalella, N., lbarzábal, D., del Valle, R., Martinez-Iglesias, J.C., ... Hernandez-Zanuy, A. (1998). Prospección del megazoobentos de los fondos blandos del Archipiélago Sabana-Camagüey, Cuba. Avicennia, 8/9, 87-104.

Alzugaray-Martínez, R., Puga-Millán, R., Piñeiro-Soto, R., de León-González, M.E., Cobas-Gómez, L.S., \& Morales-Fadragas, O. (2018). The Caribbean spiny lobster (Panulirus argus) fishery in Cuba: current status, illegal fishing, and environmental variability. Bulletin of Marine Science, 94(2), 393-408. DOI: $10.5343 / \mathrm{bms} .2016 .1126$

Arias-Schreiber, M., Wolff, M., Cano, M., MartínezDaranas, B., Marcos, Z., Hidalgo, G., ... Areces, A. (2008). Changes in benthic assemblages of the Gulf of Batabanó (Cuba): results from cruises undertaken during 1981-85 and 2003-04. Pan-American Journal of Aquatic Sciences, 3, 49-60.

Armenteros, M., Williams, J.P., Creagh, B., \& CapetilloPiñar, N. (2008). Spatial and temporal variations of meiofaunal communities from the western sector of the Gulf of Batabanó, Cuba: II. Seagrass systems. Revista de Biología Tropical, 56(1), 55-63. 
Armenteros, M., Díaz-Asencio, M., Fernández-Garcés, R., Eriksson, M., Alonso-Hernández, C., \& SanchezCabeza, J.A. (2012). Historical changes of sediments and mollusk assemblages in the Gulf of Batabanó (Caribbean Sea) in the twentieth century. Environmental Monitoring Assessment, 184, 4709-4723. DOI: $10.1007 / \mathrm{s} 10661-011-2296-\mathrm{Z}$

Baisre, J.A. 2018. Overview of Cuban commercial marine fisheries: The last 80 years. Bulletin of Marine Science, 94(2), 1-17.

Betanzos-Vega, A., Capetillo-Piñar, N., Lopeztegui-Casti1lo, A., Garcés-Rodríguez, Y., \& Tripp-Quezada, A. (2019). Parámetros meteorológicos, represamiento fluvial y huracanes. Variaciones en la hidrología del golfo de Batabanó, Cuba. Revista de Biología Marina y Oceanografia, 54(3), 308-318. DOI: 10.22370/ rbmo.2019.54.3.2024

Briones-Fourzán, P., Álvarez-Filip, L., Barradas-Ortíz, C., Morillo-Velarde, P.S., Negrete-Soto, F., SeguraGarcía, I., ... Lozano-Álvarez, E. (2019). Coral reef degradation differentially alters feeding ecology of co-occurring congeneric spiny lobsters. Frontiers in Marine Science, 5(516), 1-15.

Cerdeira-Estrada, S., Lorenzo-Sánchez, S., Areces-Mallea, A., \& Martínez-Bayón, C. (2008). Mapping of the spatial distribution of benthic habitats in the Gulf of Batabanó using Landsat-7 images. Ciencias Marinas, 34(2), 213-222.

Capetillo-Piñar, N., Villalejo-Fuerte, M.T., \& TrippQuezada, A. (2015). Distinción taxonómica de los moluscos de fondos blandos del Golfo de Batabanó, Cuba. Latin American Journal of Aquatic Research, 43(5), 856-872.

Çinar, M.E., Bakır, K., Öztürk, B., Katağan, T., Dağlı, E., Açık, Ş., ... Bitlis-Bakır, B. (2015). TUBI (TUrkish Benthic Index): A new biotic index for assessing impacts of organic pollution on benthic communities. Journal of Black Sea/Mediterranean Environment, 21(2), 135-168.

Colinas-Sánchez, F., \& Briones-Fourzán, P. (1990). Alimentación de las langostas Panulirus guttatus y $P$. argus (Latreille, 1804) en el Caribe mexicano. Anales del Instituto de Ciencias del Mar y Limnología, 17(1), 89-106

Cruz, R., Baisre, J.A., Díaz, E., Brito, R., García, C., \& Carrodeguas, C. (1990). Atlas biológico-pesquero de la langosta en el archipiélago cubano. Publicación Especial de la Revista Cubana de Investigaciones Pesqueras y la Revista Mar y Pesca.

Espinosa, J., Herrera, A., Brito, R., Diaz, E., González, G., Ibarzábal, D., \& Gotera, G. (1990). Los moluscos en la dieta de la langosta del Caribe Panulirus argus (Crustacea: Decapoda). Iberus, 9(1-2), 127-140.
Gómez, O., Ibarzábal, D., \& Silva, A. (1980). Evaluación cuantitativa de bentos en la región suroccidental de Cuba. Informe Científico-Técnico del Instituto de Oceanología, 149, 1-25.

Herrera, A., Díaz-Iglesias, E., Brito, R., González, G., Gotera, G., Espinosa, J., \& Ibarzábal, D. (1991). Alimentación natural de la langosta Panulirus argus en la región de los Indios (Plataforma SW de Cuba) y su relación con el bentos. Revista de Investigaciones Marinas, 12, 172-182.

Hidalgo, G., Toledo, W., \& Granados-Barba, A. (2015). Diversidad y distinción taxonómica de la macrofauna en fondos blandos de la plataforma norte y suroccidental cubana. Latin American Journal of Aquatic Research, 43(5), 845-855.

Martínez-Coello, D., Lopeztegui-Castillo, A., \& AmadorMarrero, U. (2015). Diferencias entre sexos en la composición de la dieta natural de la langosta Panulirus argus (Decapoda: Palinuridae) al este del golfo de Batabanó, Cuba. Research Journal of the Costa Rican Distance Education University, 7(2), 269-277.

Martínez-Daranas, B., Cano-Mallo, M., \& Clero-Alonso, L. (2009). Los pastos marinos de Cuba: estado de conservación y manejo. Serie Oceanológica, 5, 24-44.

Martínez-Daranas, B., \& Suárez, A.M. (2018). An overview of Cuban seagrasses. Bulletin of Marine Science, 94(2), 269-282. DOI: 10.5343/bms.2017.1014

Martínez-Daranas, B., Betanzos-Vega, A., Lopeztegui-Castillo, A., \& Capetillo-Piñar, N. (2018). Características del hábitat en la zona de cría de la langosta espinosa Panulirus argus al este de la Isla de la Juventud, Cuba (2010-2011). Revista de Investigaciones Marinas, $38(1), 13-32$.

McLaverty, C., Eigaard, O.R., Gislason, H., Bastardie, F., Brooks, M.E., Jonsson, P., ... Dinesen, G.E. (2020). Using large benthic macrofauna to refine and improve ecological indicators of bottom trawling disturbance. Ecological Indicators, 110, 1-13. DOI: 10.1016/j. ecolind.2019.105811

Mikkelsen, P.M., \& Bieler, R. (2007). Seashells of Southern Florida Living Marine Mollusks of the Florida Keys and Adjacent Regions: Bivalves. Hardcover, U.S.A.: Princeton University Press.

Labra, F.A., Hernández-Miranda, E., \& Quiñones, R.A. (2020). Assessing how body size affects the species-time relationship in a shallow marine benthic megafauna community exposed to a strong hypoxia disturbance. Marine Biology, 167(15), 1-13.

Long, Z.T., Bruno, J.F., \& Duffy, J.E. (2011). Food chain length and omnivory determine the stability of a marine subtidal food web. Journal of Animal Ecology, 80, 586-594. DOI: 10.1111/j.1365-2656.2010.01800.x 
Lopeztegui-Castillo, A., \& Capetillo-Piñar, N. (2008). Macrozoobentos como estimador del potencial alimentario para la langosta espinosa (Panulirus argus) en tres zonas al Sur de Pinar del Río, Cuba. Boletín del Centro de Investigaciones Biológicas, 42(2), 187-203.

Lopeztegui-Castillo, A., Capetillo-Piñar, N., \& BetanzosVega, A. (2012). Variaciones en la condición nutricional de langostas Panulirus argus (Decapoda: Palinuridae) en la región este del Golfo de Batabanó, Cuba. Revista de Biología Tropical, 60(1), 263-271.

Lozano-Álvarez, E., \& Aramoni-Serrano, G. (1996). Alimentación y estado nutricional de las langostas Panulirus inflatus y Panulirus gracilis (Decapoda: Palinuridae) en Guerrero, México. Revista de Biología Tropical, 44/45, 453-461.

Piñeiro-Soto, R., Arsenio-Areces, J., Puga-Milián, R., Cobas-Gómez, S., \& de León-González, M.E. (2017). La sustentabilidad en la pesquería de la langosta espinosa (Panulirus argus) en el golfo de Batabanó,
Cuba. II. Indicadores multidimensionales. Revista Cubana de Investigaciones Pesqueras, 34(1), 35-42.

Puga-Millán, R., Piñeiro, R., Alzugaray, R., Cobas, L.S., de León, M.E., \& Morales, O. (2013). Integrating anthropogenic and climatic factors in the assessment of the caribbean spiny lobster (Panulirus argus) in Cuba: Implications for fishery management. International Journal of Marine Science, 3(6), 36-45.

Puga-Millán, R., Valle, S., Kritzer, J.P., Delgado, G., de León, M.E., Giménez, E., Ramos, I., Moreno, O., \& Karr, K.A. (2018). Vulnerability of nearshore tropical finfish in Cuba: implications for scientific and management planning. Bulletin of Marine Science, 94(2), 1-16.

Redfern, C. (2013). Bahamian Seashells: 1161 Species from Abaco, Bahamas. Boca Raton, Florida: St. Andrews Press.

Sakshaug, E., Helge-Johnsen, G., \& Kovacs, K.M. (2009). Ecosystem Barent Sea. Trondheim, Norway: Tapir Academic Press. 